\title{
Chemoselective Union of Olefins, Organohalides and Redox-Active Es- ters Enables Regioselective Alkene Dialkylation
}

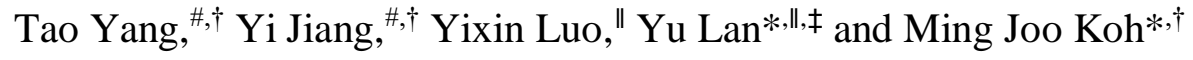 \\ 'Department of Chemistry, National University of Singapore, 12 Science Drive 2, Republic of Singapore, 117549 \\ "School of Chemistry and Chemical Engineering, Chongqing Key Laboratory of Theoretical and Computational Chemistry, \\ Chongqing University, Chongqing, 400030 \\ ${ }^{\ddagger}$ College of Chemistry and Institute of Green Catalysis, Zhengzhou University, Zhengzhou, Henan, 450001, China
}

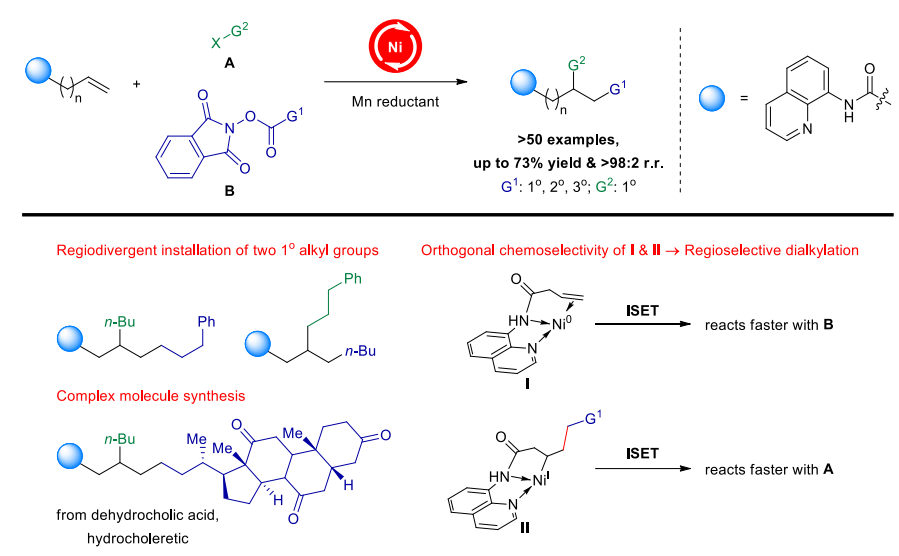

\begin{abstract}
Multicomponent catalytic processes that can generate multiple $\mathrm{C}\left(\mathrm{sp}^{3}\right)-\mathrm{C}\left(\mathrm{sp}^{3}\right)$ bonds in a single step under mild conditions, particularly if the catalysts and substrates are inexpensive, are highly sought-after in chemistry research for complex molecule synthesis. Here, we disclose an efficient Ni-catalysed reductive protocol that chemoselectively merges alkenyl amides with two different aliphatic electrophiles. Starting materials are readily accessible from stable and abundant feedstock and products are furnished in up to $>98: 2$ regioisomeric ratios. The present strategy eliminates the use of sensitive organometallic reagents, tolerates a wide array of complex functionalities and enables regiodivergent addition of two primary alkyl groups bearing similar electronic and steric attributes across aliphatic $\mathrm{C}=\mathrm{C}$ bonds with exquisite control of site selectivity. Utility is underscored by the concise synthesis of bioactive compounds and post-reaction functionalizations leading to structurally diverse scaffolds. DFT studies revealed that the regiochemical outcome originates from the orthogonal reactivity and chemoselectivity profiles of in situ-generated organonickel species.
\end{abstract}

\section{INTRODUCTION}

Transition metal-catalysed difunctionalization of unsaturated hydrocarbons represents one of the most important classes of reactions in chemistry and has extensive applications in organic synthesis. ${ }^{1}$ In this regard, a versatile set of transformations that have emerged as a powerful way through which molecular complexity can be expeditiously generated is the site-selective addition of two different carbon-based moieties across $\mathrm{C}=\mathrm{C}$ bonds. ${ }^{2}$ These reactions enable the direct incorporation of functionalities without the unnecessary intermediacy of precursors that otherwise have to be subjected to further $\mathrm{C}-\mathrm{C}$ bond-forming derivatizations. ${ }^{3}$ Notwithstanding the advances thus far, conceiving a general protocol for the regioselective insertion of $\mathrm{sp}^{3}$-hybridized carbogenic groups remains a prevailing goal in multicomponent catalytic alkene dicarbofunctionalizations. Compared to $\mathrm{sp}^{2}$-hybridized species, aliphatic substrates often exhibit attenuated reactivity (e.g. reluctance to undergo oxidative addition ${ }^{4,5}$ ) and/or are prone to undesirable side pathways (e.g. $\beta$-H elimination of the alkyl unit ${ }^{4}$ ). Reports in olefin dialkylation are scarce and often involve activated $\pi$-frameworks ${ }^{6,7}$ or two-component systems ${ }^{8}$ with organometallic nucleophiles.

An attractive approach to deliver alkyl additions to less-activated aliphatic olefins that obviates the need for unstable presynthesized alkylmetal reagents, ${ }^{9}$ relates to the direct use of organohalide electrophiles in the presence of a Ni-based catalyst and an inexpensive stoichiometric reducing agent. ${ }^{10}$ To date, high site selectivity can only be obtained when the two organohalides are electronically and/or sterically distinguishable ${ }^{11}$ (Scheme 1a). In cases where there is imperceptible bias (e.g. two primary alkyl halides), regioisomeric ratios severely diminish. As exemplified in Scheme 1b, attempts to carry out dialkyl- 
ation of olefin $4 \mathbf{a}$ with benzyl halide $\mathbf{1}$ and 1-iodobutane $\mathbf{2 a}$ under Ni-catalysed reductive conditions only led to a complex mixture of products, generating regioisomeric $\mathbf{5}$ and $\mathbf{5}$ ' in low yields and selectivity (3:1-1:1 ratio). Significant amounts of homo-dialkylation ( 6 and 7 ) and cross-electrophile coupling byproducts were also detected. We surmised that the poor regiochemical outcome stems from the inability of the catalytic organonickel species to discern the inappreciable reactivity differences between the two aliphatic halides, consequently leading to non-selective alkene insertions and mixtures of regioisomeric products.

\section{Scheme 1. The challenges in reductive dicarbofunctionali- zation of aliphatic olefins}

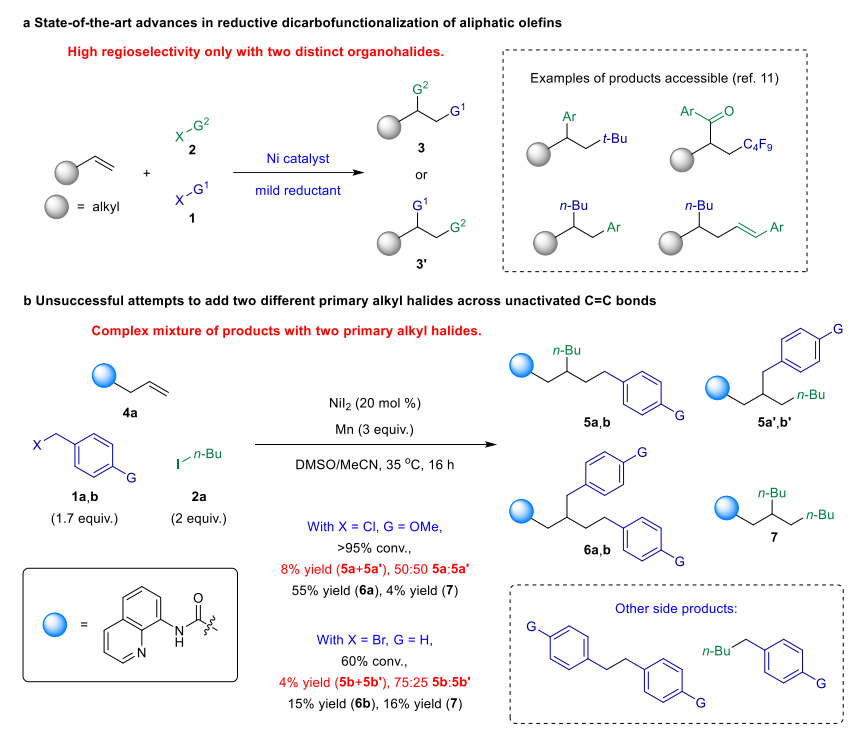

\section{RESULTS AND DISUSSION}

\section{Reaction design and mechanistic investigations}

In contemplating a plausible solution to the problem described in Scheme 1b, we wondered if electrophilic $N$ (acyloxy)phthalimides (stable and readily accessible from abundant and inexpensive carboxylic acids) ${ }^{12}$ may be leveraged as reagents to promote alkyl additions to aliphatic olefins. However, as shown in Scheme 2a, redox-active esters 9 have only been employed in catalytic difunctionalization reactions involving activated alkenes (e.g. styrenes, acrylates, trifluoromethylated olefins $)^{13}$ or 1,3 -dienes. ${ }^{14}$ Under the established conditions, $\mathbf{9}$ is prone to undergo decarboxylation through single electron transfer (SET) pathways that ultimately generate an alkyl radical $\mathbf{i}$, which is subsequently trapped by the olefin substrate in a relay process to afford ii (Scheme $2 \mathrm{a}$, dashed inset). Following catalyst recombination and further conversions, the desired difunctionalization product can be secured in high site selectivity, although only one regioisomer can be accessed in most disclosures.

Taking advantage of the susceptibility of redox-active $N$ (acyloxy)phthalimides 9 to SET processes in the presence of a nickel-based complex ${ }^{12}$ (vs. halogen atom abstraction/radical recombination with alkyl halides), we postulated that a combination of 9 and haloalkane 2 may provide the requisite reactivity differentiation for regioselective dialkyl additions to unactivated $\mathrm{C}=\mathrm{C}$ bonds in the presence of a directing auxiliary and a reductant (Scheme 2b). Successful implementation of this strategy requires that 9 first reacts with an organonickel species in the catalytic cycle, possibly generating intermediate iii that undergoes migratory insertion across the Ni-coordinated olefin, before preferentially engaging with 2 to furnish the final product $\mathbf{5}$ (or vice versa). Such a reaction trajectory (vs. the radical relay sequence in Scheme 2a) is relatively unexplored in the context of $N$-(acyloxy)phthalimide additions to alkenes. Furthermore, by reversing the aliphatic electrophiles employed (i.e. exchanging $G^{1}$ and $G^{2}$ ), access to the opposite regioisomer of 5 could be accomplished. In contrast to using bimetallic catalysis to promote orthogonal substrate activation, ${ }^{15,16}$ designing a single-catalyst system for site-selective dialkylation demands a greater degree of chemoselectivity with which the catalytic intermediates react with $\mathbf{2}$ and $\mathbf{9}$ without a large excess of either electrophilic reagent. This is more challenging to overcome compared to dialkylations that utilize distinct alkylmetal nucleophiles and haloalkane electrophiles, ${ }^{9}$ where chemoselectivity is less problematic.

Scheme 2. Design of a new catalytic regime employing haloalkanes and $N$-(acyloxy)phthalimides for site-selective dialkylation of unactivated $\mathrm{C}=\mathrm{C}$ bonds

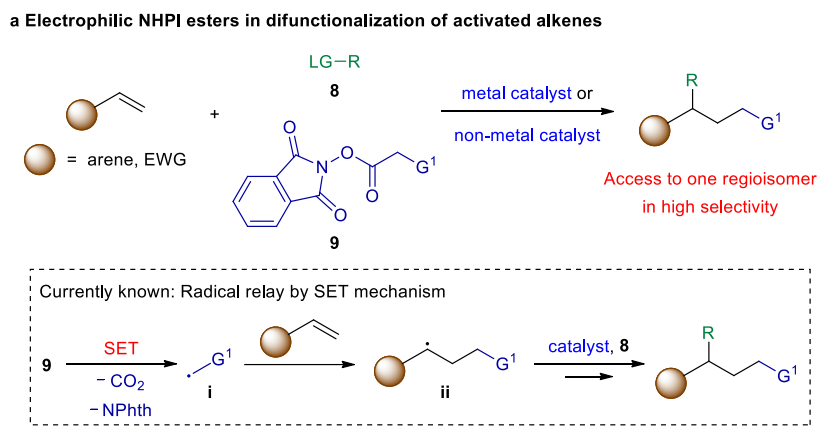

b This work: Dialkylation of unactivated alkenes with electrophilic NHPI ester \& haloalkane

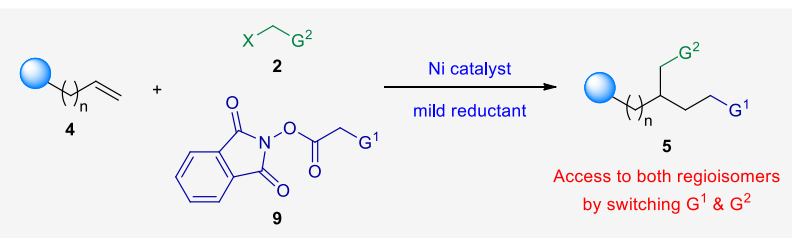

Unexplored: Intramolecular alkylnickelation

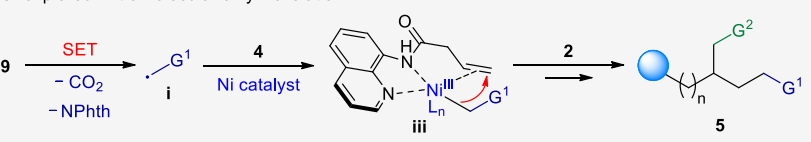

A general catalytic reductive manifold for regioselective alkene dialkylation that tolerates commonly occurring functional groups would be a significant addition to an important but limited set of transformations, facilitating the convergent synthesis of bioactive compounds and new analogues (see below for further discussion). What is more, by facile hydrolysis of the amide directing group followed by established catalytic decarboxylative reactions, ${ }^{13 c, 17,18} \mathbf{5}$ could be further converted to a wider assortment of highly functionalized compounds which might aid the development of chemical libraries for screening. Finally, the mechanistic insights derived from our studies could provide inspiration for designing new multicomponent processes that exploit the orthogonal reactivity exhibited by in situ-generated organonickel species towards different electrophiles. Herein, we disclose the details of a directed nickel-catalysed protocol that delivers site-selective dialkylation of unactivated alkenyl amides. 
Scheme 3. The underlying mechanistic principles for regioselective reductive alkene dialkylation

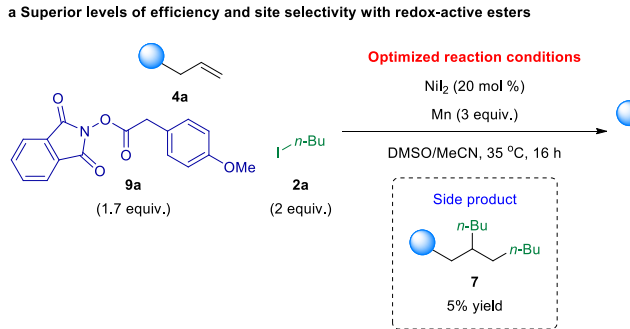

b DFT calculations
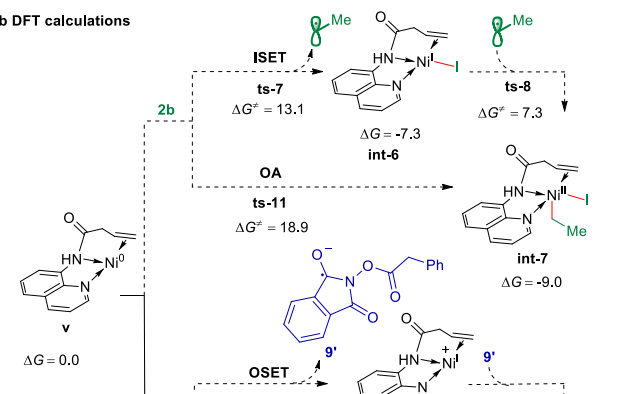

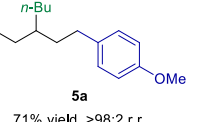

$71 \%$ yield, $>98: 2$ r.
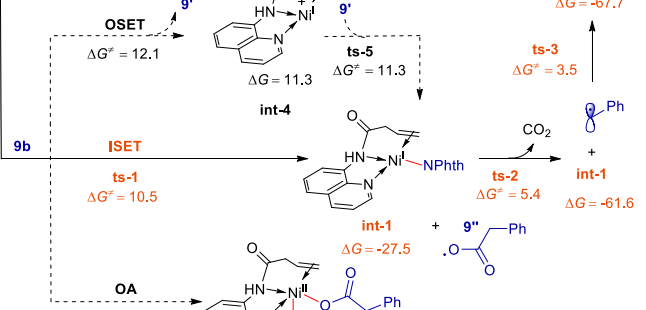

NPhth

$\Delta G=-56$

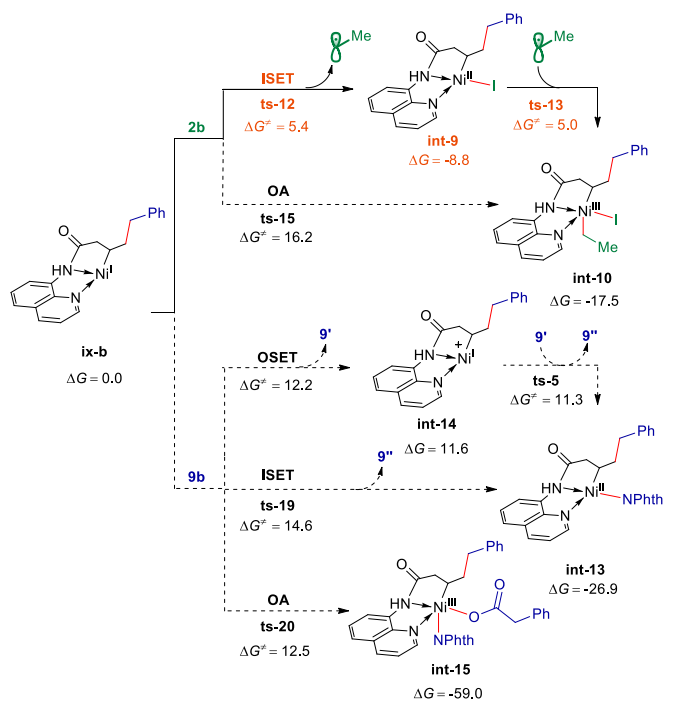

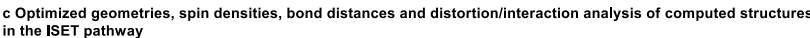

spin (Ni): 0.609

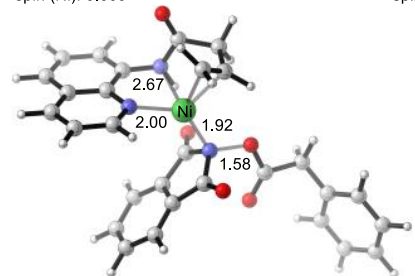

ts-1

(open-shell sing

$\Delta E_{\text {dist }}^{\neq}=37.9$

$\Delta E_{\text {int }}^{*}=-40.2$

$\Delta E_{\text {act }}^{*}=-2.3$

$\Delta E^{\neq}{ }_{\text {dist }}(\mathbf{v})=22.5$
$\Delta E^{\neq}(\mathbf{g b})=15.4$
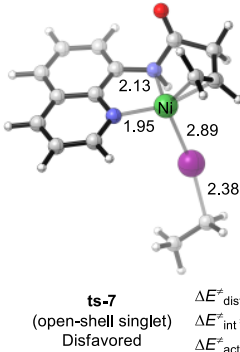

$\Delta E_{\text {dist }}^{\neq}=16.6$
$\Delta E^{*}$ int $=-12.0$

$\Delta E_{\text {act }}^{*}=4.6$

$\Delta E_{\text {dist }}^{\neq}(\mathbf{v})=12.3$

$\Delta E_{\text {dist }}^{ \pm}(\mathbf{2 b})=4.3$
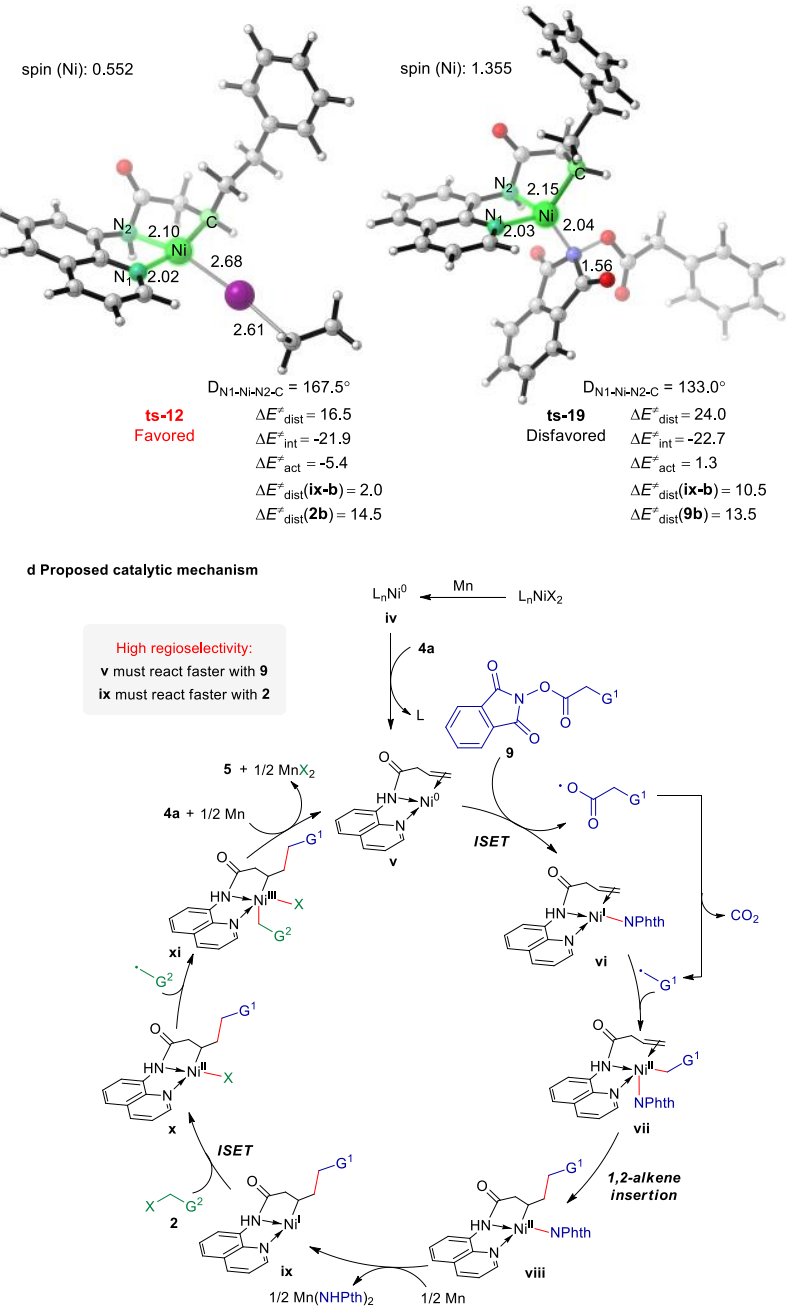

Regioisomeric ratios were determined by GC analysis with $n$-tridecane as internal standard. Yields are for isolated and purified products. The total activation energy ( $\Delta E^{\neq}$act) $=$ distortion energy $\left(\Delta E_{\text {dist }}^{\neq}\right)+$interaction energy $\left(\Delta E^{\neq}\right.$int $)$. The distortion energy consists of two reacting partner terms $\left[\Delta E^{\neq}{ }_{\text {dist }}(\right.$ a $\left.)+\Delta E_{\text {dist }}^{\neq}(\mathrm{b})\right]$. All energies are reported in kcal/mol. All bond distances are reported in ångstrom. G, functional group; X, halide; Phth, phthaloyl; DMSO, dimethyl sulfoxide; NMP, $N$-methyl-2-pyrrolidone; OA, oxidative addition; ISET, inner-sphere single electron transfer; OSET, outer-sphere single electron transfer; int, intermediate; ts, transition state; $\Delta G$, Gibbs free energy; $\Delta G G^{\neq}$, Gibbs free energy of activation; $\Delta E_{\text {act }}^{\neq}$, total activation energy; $\Delta E_{\text {dist, }}^{\neq}$distortion energy; $\Delta E_{\text {int, }}^{\neq}$interaction energy; spin, spin densities; D, dihedral angle.

We commenced our studies by examining conditions that promote the union of directing group-tethered aliphatic olefins with 2a and $N$-(acyloxy)phthalimide 9a. After an extensive evaluation of conditions including directing auxiliaries, Nibased salts, solvents and reducing agents (see Tables S1-4 for details), best results were secured when 8 -aminoquinoline-tethered alkene 4a was merged with 2a and 9a in the presence of $20 \mathrm{~mol} \% \mathrm{NiI}_{2}$ and 3 equiv. $\mathrm{Mn}$ in DMSO/MeCN, furnishing $\mathbf{5 a}$ in $71 \%$ yield and complete site selectivity with trace amounts of homo-dialkylation by-product (Scheme 3a). This unexpected outcome is a remarkable improvement compared to the analogous reaction using alkyl halides in which $\mathbf{5 a}$ was generated in poor yield as a 50:50 regioisomeric mixture with significant formation of side products (cf. Scheme 1b). 
Based on the above experimental observations, density functional theory (DFT) method M06-L ${ }^{19}$ was employed to investigate the mechanistic nuances that account for the orthogonal reactivity of the two electrophiles in the Ni-catalysed reductive alkene dialkylation (Scheme 3b). In the presence of $\mathrm{Mn}$, nickel(II) salts may be reduced to $\mathrm{Ni}(0)$ species, ${ }^{10 a, 10 \mathrm{~g}}$ which could associate with 8-aminoquinoline-tethered olefin $4 \mathbf{4 a}$ to generate $\mathrm{Ni}(0)$ complex $\mathbf{v}$, the key species to be considered in the following processes. Under the reaction conditions, either $N$-(acyloxy)phthalimide $\mathbf{9 b}$ or iodoethane $\mathbf{2 b}$ (model substrates) could serve as the oxidant to react with $\mathbf{v}$. Specifically, the competition between these two electrophilic reagents in the $\mathrm{Ni}$ oxidation steps will influence the regiochemical outcome of the olefin dialkylation. In our theoretical study, three types of oxidative mechanisms ${ }^{20}$ involving inner-sphere single electron transfer (ISET), outer-sphere single electron transfer (OSET) and concerted oxidative addition (OA) were examined for the reaction of $\mathbf{9 b}$ or $\mathbf{2 b}$ with different $\mathrm{Ni}$ species. When $\mathbf{2} \mathbf{b}$ was used as oxidant to react with $\mathrm{Ni}(0)$ complex $\mathbf{v}$, the calculated free energy barrier for the ISET process was $13.1 \mathrm{kcal} / \mathrm{mol}$ via an ISET-assisted homolytic $\mathrm{C}-\mathrm{I}$ bond cleavage transition state ts-7 leading to the formation of a Ni(I)-iodide species int-6 and an ethyl radical. Subsequent recombination with ethyl radical furnishes $\mathrm{Ni}$ (II)-alkyl intermediate int-7. Although intermediate int-7 could also be generated from a concerted OA process via three-membered ring transition state ts-11, such a pathway could be excluded by the relatively higher free energy barrier of $18.9 \mathrm{kcal} / \mathrm{mol}$.

Alternatively, when 9b undergoes reaction with $\mathbf{v}$, DFT calculations revealed that the free energy barrier for the ISET pathway (homolytic N-O bond dissociation) is only $10.5 \mathrm{kcal} / \mathrm{mol}$ via transition state ts-1. In this process, the coordination of phthalimide nitrogen to the $\mathrm{Ni}$ center results in $\mathrm{N}-\mathrm{O}$ bond cleavage in $\mathbf{9 b}$, generating a $\mathrm{Ni}(\mathrm{I})$-phthalimide species int-1 with concomitant release of a phenylacetate radical 9". Facile decarboxylation of 9" ejects a benzyl radical, which can further oxidize int-1 via transition state ts-3 to afford Ni(II)-benzyl intermediate int-2. In contrast, the free energy barrier for the concerted OA pathway was found to be much higher $(20.7 \mathrm{kcal} / \mathrm{mol})$ than that of the ISET process. On the other hand, $\mathbf{9 b}$ may serve as a single electron acceptor and participate in a OSET process as commonly proposed in the literature. ${ }^{12}$ The calculated activation free energy for this pathway by using approximation of modified Marcus theory ${ }^{21}$ is $12.1 \mathrm{kcal} / \mathrm{mol}$ to deliver a cationic $\mathrm{Ni}(\mathrm{I})$ intermediate int-4 that is $11.3 \mathrm{kcal} / \mathrm{mol}$ endergonic. However, after the ensuing phthalimide anion transfer to form int-1 via ts-5, the calculated activation free energy of the overall process is as high as $22.6 \mathrm{kcal} / \mathrm{mol}$. Thus, the OSET pathway is likely to be unfavorable.

On the basis of the above calculations, $N$ (acyloxy)phthalimide $\mathbf{9 b}$ is found to chemoselectively react with $\mathrm{Ni}(0)$ complex $\mathbf{v}$ through stepwise ISET-type oxidation, contrary to previously established pathways, to afford $\mathrm{Ni}$ (II)benzyl intermediate int-2. Following intramolecular 1,2-alkene insertion into the $\mathrm{Ni}-\mathrm{C}$ (benzyl) bond and single electron reduction by $\mathrm{Mn}$, a Ni(I)-alkyl species ix-b is formed, which can subsequently react with either $\mathbf{9 b}$ or iodoethane $\mathbf{2 b}$. Further DFT studies indicated that ix-b readily reacts with $\mathbf{2 b}$ through a ISET process via transition state ts-12 with an activation free energy of only $5.4 \mathrm{kcal} / \mathrm{mol}$. The resulting Ni(II)-iodide int-9 then captures the ethyl radical to furnish $\mathrm{Ni}$ (III)-dialkyl intermediate int-10, which is susceptible to reductive elimination to yield the desired dialkylation product.

\section{Scheme 4. Radical clock studies}
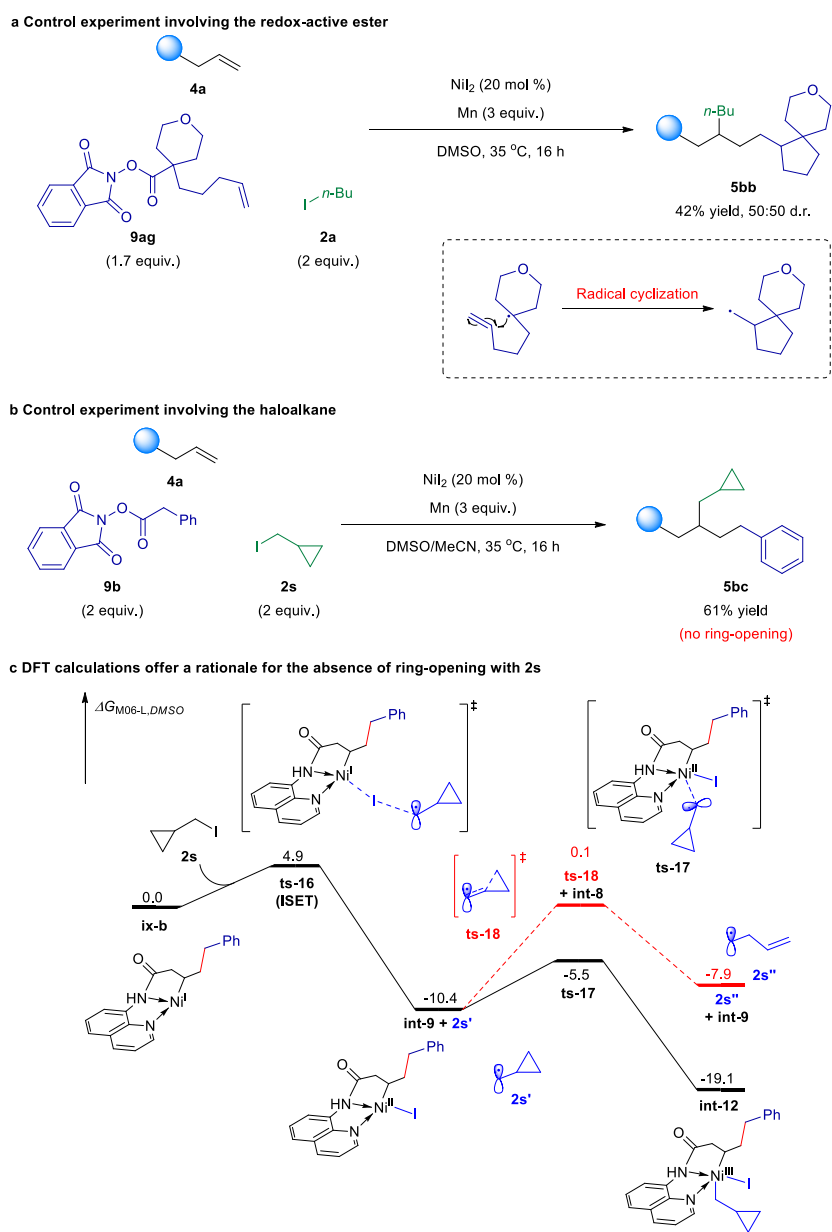

For comparison, we also evaluated the reactivity profile of ix-b with $N$-(acyloxy)phthalimide $9 \mathbf{b}$. The calculated free energy barrier for the ISET pathway is $14.6 \mathrm{kcal} / \mathrm{mol}$ via transition state ts-19, which is $9.2 \mathrm{kcal} / \mathrm{mol}$ higher than that with $\mathbf{2 b}$ via ts-12. Furthermore, the corresponding OSET and OA pathways were also found to be energetically less favorable compared to the calculated ISET pathway with $\mathbf{2} \mathbf{b}$. Therefore, the excellent site selectivity that was observed in Scheme 3 a primarily arose from the orthogonal reactivity of $N$-(acyloxy)phthalimide $9 \mathbf{b}$ and iodoethane $\mathbf{2 b}$ with different organonickel species (i.e. $\mathbf{v}$ and $\mathbf{i x - b}$ ) generated in the catalytic system.

\section{Scheme 5. The scope of redox-active esters in catalytic reductive dialkylation ${ }^{a}$}




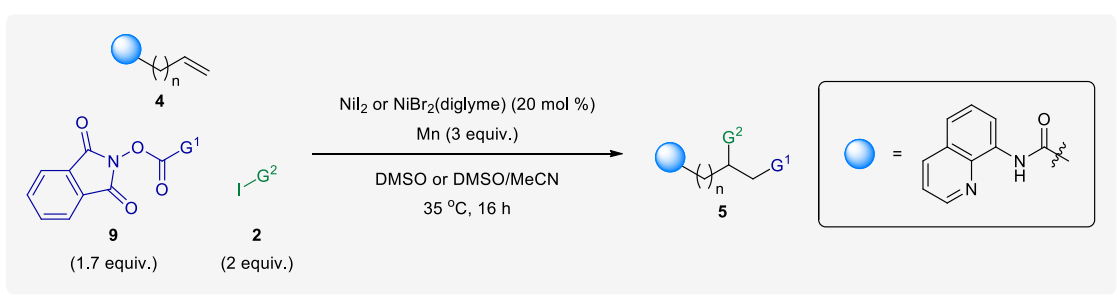

Primary alkyl NHPI esters

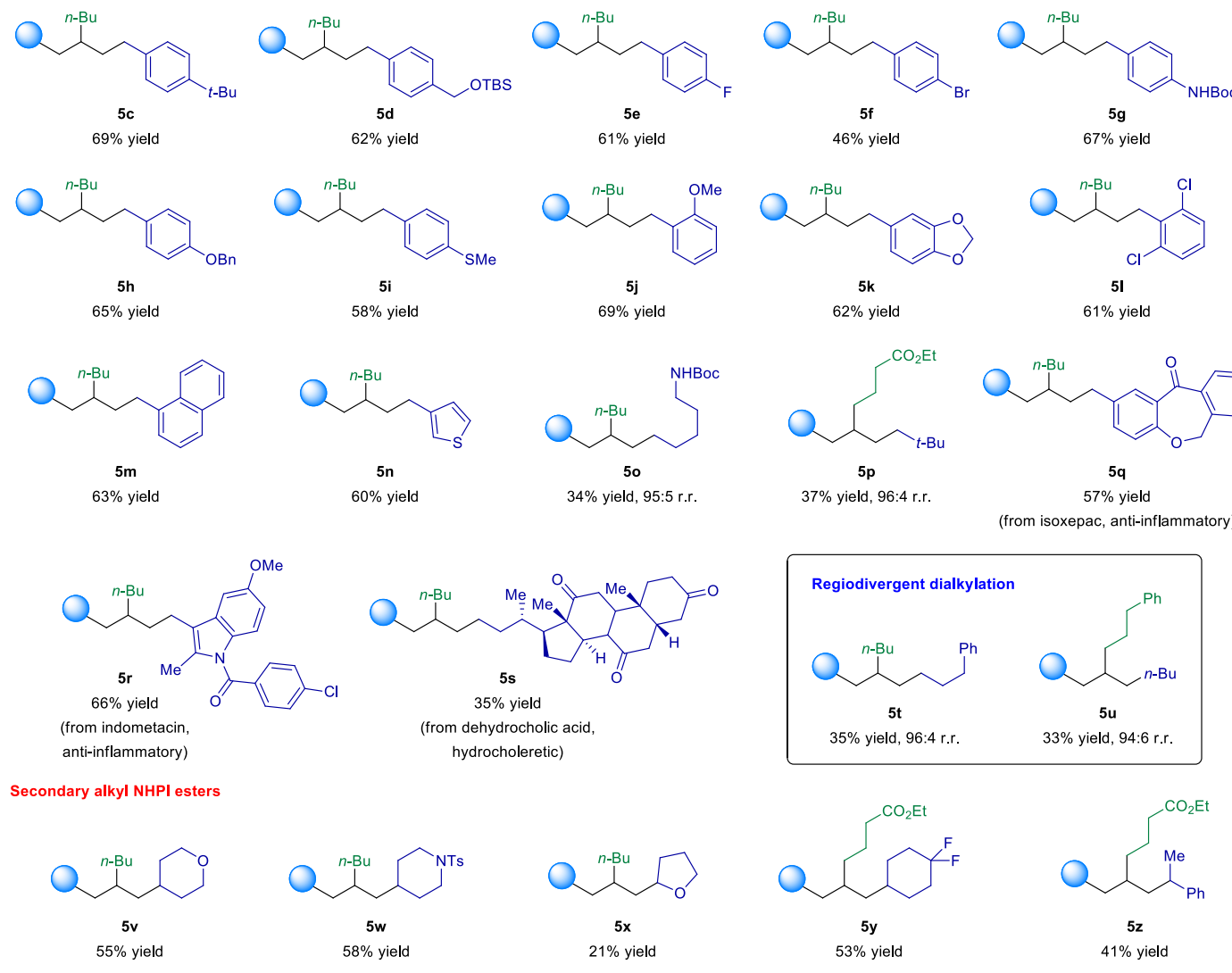

Tertiary alkyl NHPI esters
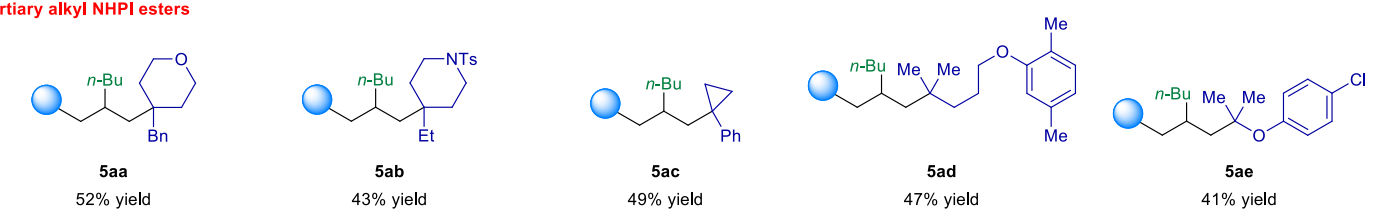

$\gamma, \delta$-Unsaturated amides
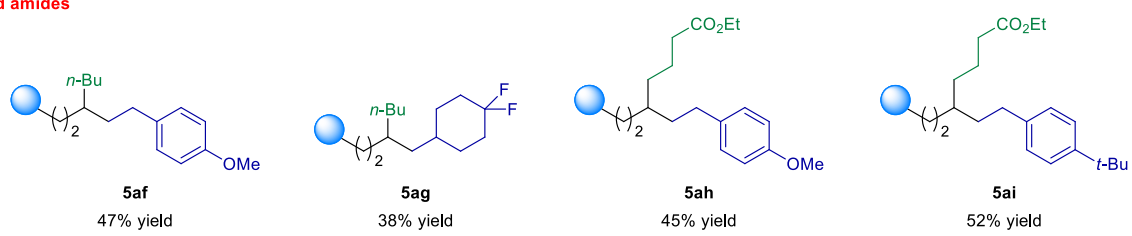

aUnless otherwise stated, all dialkylation products were obtained as single regioisomers. Regioisomeric ratios were determined by GC analysis with $n$-tridecane as internal standard. Yields are for isolated and purified products. $\mathbf{5}$ and $\mathbf{5 x}$ were both obtained as 1:1 diastereomeric mixtures. 5z was obtained as a 1.4:1 diastereomeric mixture. For $\mathbf{5 x}-\mathbf{5 z}$, NHPI ester (2.0 equiv.) was used. For $\mathbf{5 a a}, \mathbf{5 a f}, \mathbf{5 a h}$ and $\mathbf{5 a i}, \mathbf{N i}(\operatorname{cod})_{2}$ was used as catalyst. For 5af-5ai, NMP was used as solvent and NHPI ester (2.5 equiv.) was used. See Supporting Information for details.

The origin of the high regioselectivity cannot be simply rationalized by conventional electronic and/or steric arguments involving the two electrophiles and the in situ-generated $\mathrm{Ni}$ complexes $\mathbf{v}$ and $\mathbf{i x}-\mathbf{b}$. To gain further insights, careful distortion/interaction analysis ${ }^{22}$ of the four ISET-type transition states ts-1, ts-7, ts-12, and ts-19 was carried out, where the total activation energy ( $\Delta E_{\text {act }}^{*}$ ) was separated into the distortion energy of two reacting partners $\left(\Delta E^{\neq}\right.$dist $)$and the interaction energy between those two distorted partners $\left(\Delta E^{\neq}\right.$int $)$. As shown in
Scheme 3c, when $N$-(acyloxy)phthalimide $9 \mathbf{b}$ reacts with $\mathrm{Ni}(0)$ species $\mathbf{v}$, a large interaction energy of $-40.2 \mathrm{kcal} / \mathrm{mol}$ was observed in transition state ts-1. This can be explained by the strong association of the phthalimide nitrogen with the Ni center. The distortion energy of ts-1 largely arises from dissociation of the 8-aminoquinoline amide nitrogen, which may be nullified by the coordination of $\mathbf{9 b}$. On the contrary, the interaction between iodoethane $\mathbf{2 b}$ and $\mathbf{v}$ in transition state ts-7 is significantly lower. Hence, $\Delta E^{\neq}$act becomes more favorable in ts-1 (vs. ts-7) and $\mathbf{v}$ is predicted to preferentially undergo oxidation 
with $\mathbf{9 b}$ (instead of $\mathbf{2 b}$ ). Intriguingly, the interaction between $\mathbf{2 b}$ and the more electron-deficient $\mathrm{Ni}(\mathrm{I})$ species ix-b is dramatically enhanced in transition state ts-12, whereas the distortion energy remains almost unchanged (vs. ts-7). On the other hand, the association of $\mathbf{i x}-\mathbf{b}$ with $\mathbf{9 b}$ causes greater distortion within ts-19 (D, dihedral angle; $\mathrm{D}_{\mathrm{N} 1-\mathrm{Ni}-\mathrm{N} 2-\mathrm{C}}=133.0^{\circ}$ vs. reaction with $2 b$ via ts-12, $\left.\mathrm{D}_{\mathrm{N} 1-\mathrm{Ni}-\mathrm{N} 2-\mathrm{C}}=167.5^{\circ}\right)$. Therefore, the second oxidation involving ix-b would selectively occur in the presence of $\mathbf{2 b}$ (instead of $\mathbf{9 b}$ ).

Our DFT results are supported by control experiments in which both the $N$-(acyloxy)phthalimide and alkyl halide reagent likely reacted through ISET-type radical processes (Scheme $4 \mathrm{a}, \mathrm{b})$. Although the use of (iodomethyl)cyclopropane 2s delivered the expected product $\mathbf{5 b c}$ with no trace of ring rupturing, computations showed that the ring-opening rate of the cyclopropylmethyl radical intermediate (generated from iodine abstraction/radical recombination) is comparatively slower than the rate of radical association with the catalytic organonickel species (Scheme 4c). This result is in contrast to a previous dialkylation disclosure in which only the ring-cleaved product was detected. ${ }^{9}$

Consolidating the information derived from experimental and theoretical studies, we propose a possible catalytic cycle (Scheme 3d) that begins with initial formation of a putative $\mathrm{Ni}(0)$ species iv (possibly from reduction of the $\mathrm{Ni}(\mathrm{II})$ pre-catalyst by $\mathrm{Mn})^{10 \mathrm{a}, 10 \mathrm{~g}}$ that undergoes ligand exchange with substrate 4a to generate $\mathrm{Ni}(0)$ complex $\mathbf{v}$. Instead of engaging with the alkyl halide $\mathbf{2}, \mathbf{v}$ chemoselectively reacts with the $N$ (acyloxy)phthalimide $\mathbf{9}$ through an ISET process to afford a $\mathrm{Ni}(\mathrm{I})$-phthalimide intermediate vi and a carboxylate radical. Facile extrusion of $\mathrm{CO}_{2}$ furnishes an alkyl radical that recombines with vi to give $\mathrm{Ni}(\mathrm{II})$-alkyl intermediate vii. An intramolecular site-selective alkylnickelation across the chelated $\pi$ bond to form viii followed by reduction of the Ni center by $\mathrm{Mn}$ could proceed to generate $\mathrm{Ni}(\mathrm{I})$-alkyl complex ix. At this stage, ix preferentially reacts with 2 through another ISET pathway to deliver a new Ni(III)-alkyl species xi (via $\mathbf{x}$ ) that reductively eliminates to release the desired dialkylation adduct $\mathbf{5}$, before an ensuing reduction process and ligand exchange regenerate $\mathbf{v}$.

\section{Substrate scope and synthetic applications}

We first surveyed the reaction scope by merging different classes of aliphatic $N$-(acyloxy)phthalimides 9 with alkene 4 and iodoalkane 2 under the optimized conditions (Scheme 5). A wide assortment of primary alkyl-substituted redox-active esters underwent reductive dialkylation, affording the desired adducts $\mathbf{5 c - 5 p}$ in $34-69 \%$ yield and $\geq 95: 5$ regioisomeric ratios. These include products containing heterocycles $(\mathbf{5 k}, \mathbf{5 n})$ as well as a halogen motif (5f) and a protic $\mathrm{N}-\mathrm{H}$ group $(\mathbf{5 g}, \mathbf{5 o})$ that might otherwise react if the corresponding alkylmetal reagent is utilized. ${ }^{23}$

$N$-(Acyloxy)phthalimides derived from multifunctional and biologically active carboxylic acids also reacted to deliver $\mathbf{5 q}-\mathbf{5 r}$, further underscoring the method's exceptional functional group tolerance. Yields were somewhat diminished when reagents bearing long-chain alkyl units were involved (5o-5p and $\mathbf{5} \mathbf{s}-\mathbf{5 u}$ ) owing to competitive formation of homo-dialkylation, cross-electrophile coupling and other side products (see Tables S5-11 for details). Nevertheless, the catalytic protocol is amenable to the selective generation of either regioisomer (5t-5u) simply by exchanging $G^{1}$ and $G^{2}$ moieties within 9 and 2.
Reactions with secondary and tertiary alkyl-substituted $\mathrm{N}$ (acyloxy)phthalimides were similarly effective to deliver regioisomerically pure products $\mathbf{5 v}-\mathbf{5 a e}$ in up to $58 \%$ yield. These comprise of molecules that bear heteroatom-substituted $(\mathbf{5} \mathbf{x}$, $\mathbf{5 a e})$ and all-carbon quaternary (5aa-5ad) stereogenic centers, offering a broader range of adducts compared to a previous protocol involving largely primary alkyl additions with organozinc reagents. ${ }^{9}$ For reactions that employed tertiary alkyl redox-active esters, DFT calculations suggested that both intramolecular alkylnickelation (cf. Scheme $2 \mathrm{~b}$ ) and radical relay-based process (cf. Scheme 2a) are energetically comparable, which means that either pathway may be operative under the reaction conditions to deliver the same sense of regioselectivity (see Fig. S2 for details). Extending the carbon-chain length to $\gamma, \delta$-unsaturated alkenyl amides did not appreciably affect dialkylation as the corresponding adducts $\mathbf{5 a f}-\mathbf{5 a i}$ could be generated in $38-52 \%$ yield. However, substrates with $1,1-$ or 1,2-disubstituted $\mathrm{C}=\mathrm{C}$ bonds were unreactive ( $<5 \%$ conv. to product).

Scheme 6. The scope of aliphatic halides in catalytic reductive dialkylation ${ }^{\mathrm{a}}$
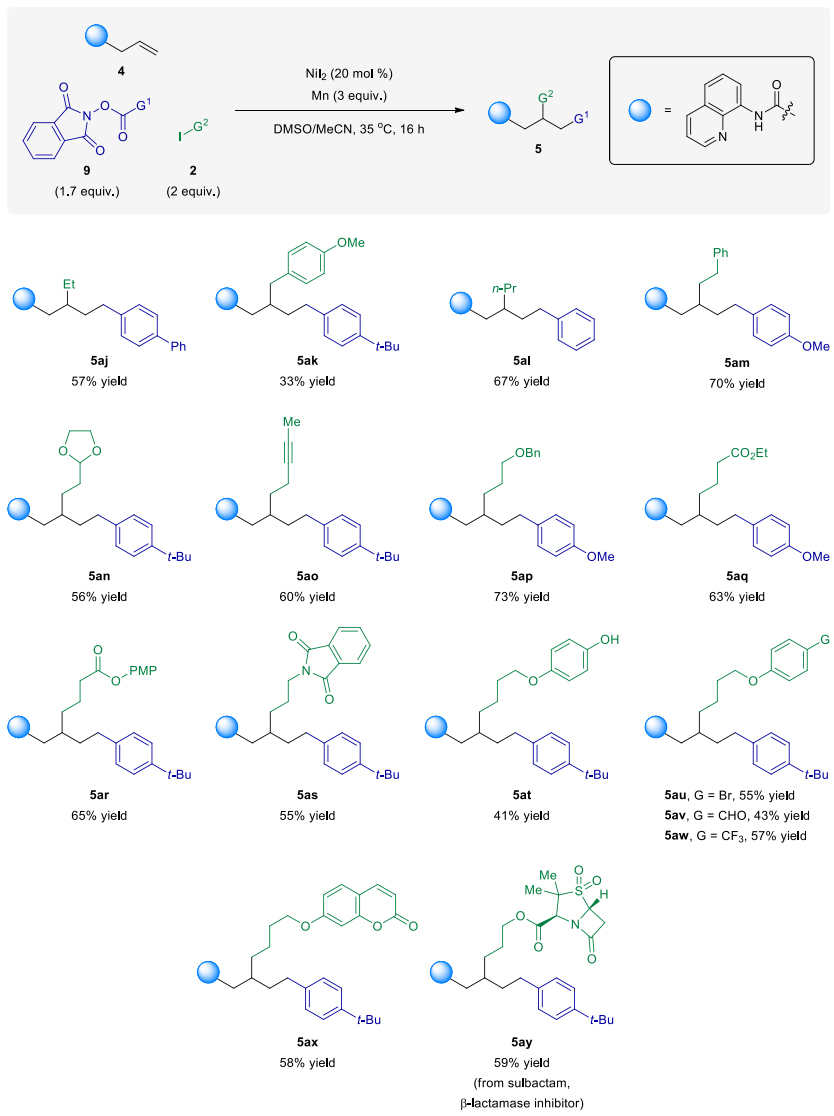

${ }^{a}$ All dialkylation products were obtained as single regioisomers. Yields are for isolated and purified products. For $\mathbf{5} \mathbf{a k}$ and $\mathbf{5} \mathbf{a t}, \mathrm{NiBr}_{2}$ (diglyme) was used as catalyst. For $\mathbf{5} \mathbf{a k}$, 4-methoxybenzyl chloride was used as reagent and DMPU was used as solvent. See Supporting Information for details.

The nature of the alkyl iodide $\mathbf{2}$ could also be varied under the optimized reductive dialkylation conditions (Scheme 6). Various functionalized products 5aj-5ay, including those that bear an acid-labile acetal (5an), an alkyne (5ao), carboxylic esters (5aq-5ar), a Lewis basic phthalimide (5as), a Brønsted acidic phenol (5at), an aldehyde (5av) as well as a cyclic enoate (5ax) could be secured in up to $73 \%$ yield as single regioisomers. As highlighted by the formation of 5ay, the catalytic method is also compatible with reagents derived from complex 
molecules such as $\beta$-lactamase inhibitor sulbactam, whose sensitivity to alkaline conditions ${ }^{24}$ might pose problems with basic organometallic reagents.

The ability to construct two $\mathrm{C}\left(\mathrm{sp}^{3}\right)-\mathrm{C}\left(\mathrm{sp}^{3}\right)$ bonds in one step with exceptional site selectivity under mild reaction conditions presents an opportunity to devise new synthesis routes for a number of important compounds of interest (Scheme 7a). In one instance, amide deprotection of the dialkylation product 5az (synthesized on $2 \mathrm{mmol}$ scale) led to the known acid 10a, a precursor of antibiotic potentiator 11a, in $51 \%$ overall yield. The three-step sequence from commercially available starting materials is more concise than a previous five-step route. ${ }^{25}$ Furthermore, this approach potentially allows new substituted analogues to be more readily accessed for evaluation of medicinal properties by merging different combinations of $\mathrm{N}$ (acyloxy)phthalimides and alkyl halides. An exemplary case relates to the facile preparation of methoxy-derivative 11b, which may be generated from 5 am via $\mathbf{1 0 b}$.

Scheme 7. Access to medicinally relevant molecules and further transformations ${ }^{\mathrm{a}}$

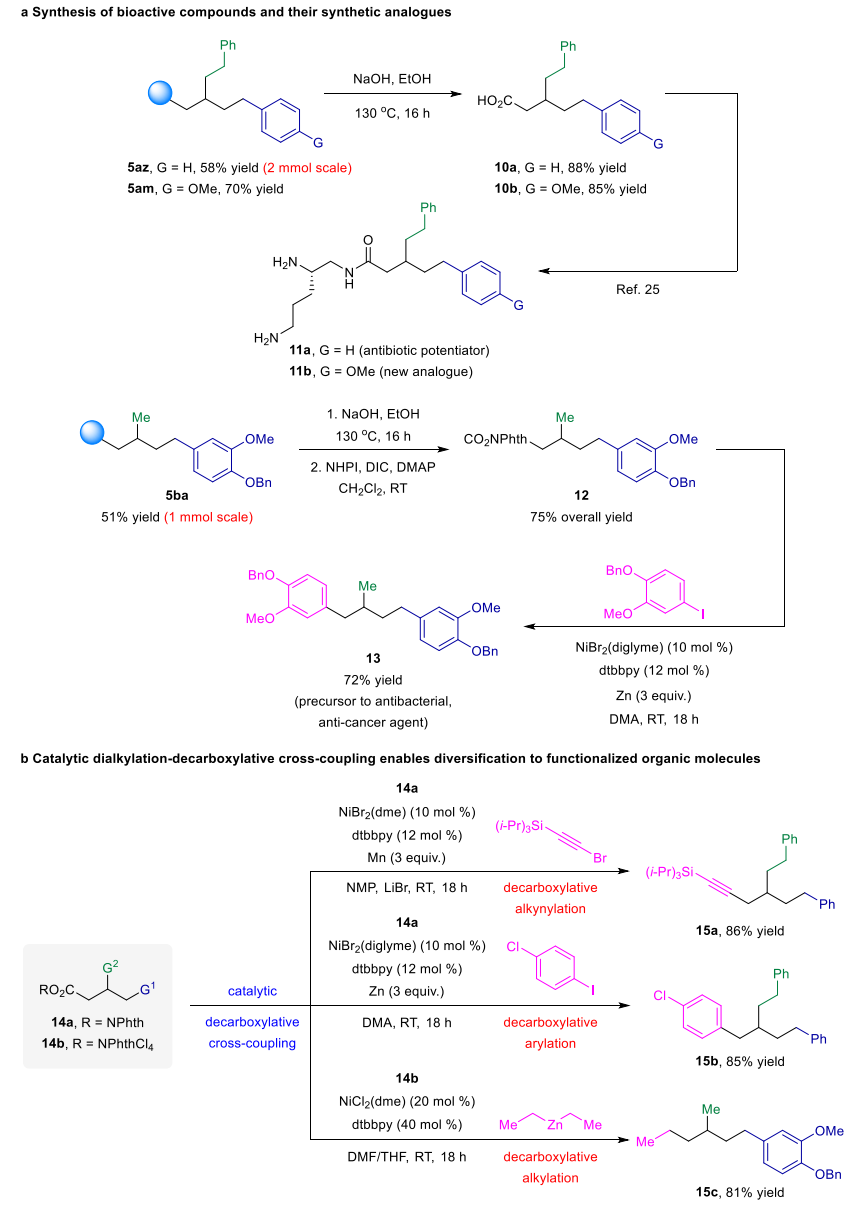

${ }^{a}$ All dialkylation products were obtained as single regioisomers. Yields are for isolated and purified products. See Supporting Information for details.

The second application involves the preparation of $\mathbf{1 3}$, an intermediate employed in the synthesis of a compound with antibacterial and anti-cancer activities. ${ }^{26}$ Conversion of $\mathbf{5 b a}$ (synthesized on $1 \mathrm{mmol}$ scale) to the corresponding redox-active ester 12 followed by Ni-catalysed reductive cross-coupling ${ }^{17}$ with 1-(benzyloxy)-4-iodo-2-methoxybenzene delivered 13 in 54\% yield over three steps. A similar decarboxylative strategy was adopted to afford a variety of different analogues (see Supporting Information Section $2.4 \mathrm{C}$ for details).
As shown in Scheme 7b, the appropriate combination of catalytic reductive dialkylation of unsaturated amides and catalytic decarboxylative cross-coupling can enable multiple carbon-carbon linkages to be installed in a regioselective fashion, thus providing an expedient avenue to create complexity and diversity for compound library synthesis. To further illustrate this versatility, $N$-(acyloxy)phthalimides derived from dialkylation adducts were subjected to established decarboxylative transformations ${ }^{13 c, 17,18}$ (furnishing $\mathrm{C}(\mathrm{sp})-\mathrm{C}\left(\mathrm{sp}^{3}\right), \mathrm{C}\left(\mathrm{sp}^{2}\right)-\mathrm{C}\left(\mathrm{sp}^{3}\right)$ and $\mathrm{C}\left(\mathrm{sp}^{3}\right)-\mathrm{C}\left(\mathrm{sp}^{3}\right)$ bonds) to give $\mathbf{1 5 a}-\mathbf{c}$ in $81-86 \%$ yield (Scheme $7 \mathrm{~b})$.

\section{CONCLUSIONS}

By leveraging the differences in reactivity and chemoselectivity patterns of in situ-generated organonickel species, we have successfully developed a directed $\mathrm{Ni}$-catalysed manifold that efficiently merges unactivated alkenyl amides with haloalkanes and aliphatic redox-active esters under reductive conditions. This approach avoids the use of sensitive organometallic reagents and overcomes the longstanding challenge of installing two primary alkyl units, derived from stable electrophilic reagents, across $\mathrm{C}=\mathrm{C}$ bonds with remarkable regioselectivity. The present protocol's capability to forge multiple $\mathrm{C}-\mathrm{C}$ bonds between $\mathrm{sp}^{3}$-hybridized moieties that constitute the skeletal backbone of organic molecules, ${ }^{27}$ along with the relative ease of product derivatization, offer a direct entry to multifunctional entities. The insights gained from these studies are expected to aid future development of reactions that involve multiple electrophiles for the assembly of important compounds of interest.

\section{ASSOCIATED CONTENT}

\section{Supporting Information}

This material is available free of charge via the Internet at http://pubs.acs.org.

Experimental procedures and analytical data for all compounds, DFT calculations

\section{AUTHOR INFORMATION}

\section{Corresponding Authors}

*Ming Joo Koh - Department of Chemistry, National University of Singapore, 12 Science Drive 2, Republic of Singapore, 117549; ORCID: 0000-0002-2534-4921; Email: chmkmj@nus.edu.sg.

*Yu Lan - School of Chemistry and Chemical Engineering, Chongqing Key Laboratory of Theoretical and Computational Chemistry, Chongqing University, Chongqing, 400030, China; College of Chemistry and Institute of Green Catalysis, Zhengzhou University, Zhengzhou, Henan, 450001, China; ORCID: 00000002-2328-0020; Email: lanyu@cqu.edu.cn.

\section{Authors}

\#Tao Yang - Department of Chemistry, National University of Singapore, 12 Science Drive 2, Republic of Singapore, 117549.

\#Yi Jiang - Department of Chemistry, National University of Singapore, 12 Science Drive 2, Republic of Singapore, 117549.

Yixin Luo - School of Chemistry and Chemical Engineering, Chong-qing Key Laboratory of Theoretical and Computational Chemistry, Chongqing University, Chongqing, 400030, China. 


\section{Author Contributions}

${ }^{\#}$ T.Y. and Y.J. contributed equally.

\section{Notes}

The authors declare no competing financial interests.

\section{ACKNOWLEDGMENT}

This research was supported by the National University of Singapore Academic Research Fund Tier 1: R-143-000-A77-114 (M.J.K.) and the 111 Project: D20003 (Y.L.).

\section{REFERENCES}

(1) For selected reviews, see: (a) McDonald, R. I.; Liu, G. S.; Stahl, S. S. Palladium(II)-catalyzed alkene functionalization via nucleopalladation: stereochemical pathways and enantioselective catalytic applications. Chem. Rev. 2011, 111, 2981-3019. (b) Beller, M.; Seayad, J.; Tillack, A.; Jiao, H. Catalytic markovnikov and anti-markovnikov functionalization of alkenes and alkynes: recent developments and trends. Angew. Chem. Int. Ed. 2004, 43, 3368-3398. (c) Yin, G.; Mu, X.; Liu, G. Palladium(II)-catalyzed oxidative difunctionalization of alkenes: bond forming at a high-valent palladium center. Acc. Chem. Res. 2016, 49, 2413-2423.

(2) For selected reviews and examples, see: (a) Dhungana, R. K.; KC, S.; Basnet, P.; Giri, R. Transition metal-catalyzed dicarbofunctionalization of unactivated olefins. Chem. Rec. 2018, 18, 1314-1340. (b) Derosa, J.; Apolinar, O.; Kang, T.; Tran, V. T.; Engle, K. M. Recent developments in nickel-catalyzed intermolecular dicarbofunctionalization of alkenes. Chem. Sci. 2020, 11, 4287-4296. (c) Qi, X.; Diao, T. Nickel-catalyzed dicarbofunctionalization of alkenes. ACS Catal. 2020, 10, 8542-8556. (d) Zhang, J.-S.; Liu, L.; Chen, T.; Han, L.-B. Transition-metal catalyzed three-component difunctionalizations of alkenes. Chem. Asian J. 2018, 13, 2277-2291. (e) Liao, L.; Jana, R.; Urkalan, K. B.; Sigman, M. S. A palladium-catalyzed three-component cross coupling of conjugated dienes or terminal alkenes with vinyl triflates and boronic acids. J. Am. Chem. Soc. 2011, 133, 5784-5787. (f) Gao, P.; Chen, L. A.; Brown, M. K. Nickel-catalyzed stereoselective diarylation of alkenylarenes. J. Am. Chem. Soc. 2018, 140, 10653-10657. (g) Shrestha, B.; Basnet, P.; Dhungana, R. K.; KC, S.; Thapa, S.; Sears, J. M.; Giri, R. Ni-Catalyzed regioselective 1,2-dicarbofunctionalization of olefins by intercepting Heck intermediates as iminestabilized transient metallacycles. J. Am. Chem. Soc. 2017, 139, 10653-10656. (h) Derosa, J.; Tran, V. T.; Boulous, M. N.; Chen, J. S.; Engle, K. M. Nickel-catalyzed $\beta, \gamma$-dicarbofunctionalization of alkenyl carbonyl compounds via conjunctive cross-coupling. J. Am. Chem. Soc. 2017 , 139, 10657-10660. (i) Gu, J. W.; Min, Q. Q.; Yu, L. C.; Zhang, X. G. Tandem difluoroalkylation-arylation of enamides catalyzed by nickel. Angew. Chem. Int. Ed. 2016, 55, 12270-12274. (j) KC, S.; Dhungana, R.; Shrestha, B.; Thapa, S.; Khanal, N.; Basnet, P.; Lebrun, R. W.; Giri, R. Ni-Catalyzed regioselective alkylarylation of vinylarenes via $\mathrm{C}\left(\mathrm{sp}^{3}\right)-\mathrm{C}\left(\mathrm{sp}^{3}\right) / \mathrm{C}\left(\mathrm{sp}^{3}\right)-\mathrm{C}\left(\mathrm{sp}^{2}\right)$ bond formation and mechanistic studies. $J$. Am. Chem. Soc. 2018, 140, 9801-9805. (k) Chierchia, M.; Xu, P.; Lovinger, G. J.; Morken, J. P. Enantioselective radical addition/cross-coupling of organozinc reagents, alkyl iodides, and alkenyl boron reagents. Angew. Chem. Int. Ed. 2019, 58, 14245-14249. (1) Derosa, J.; Kleinmans, R.; Tran, V. T.; Karunananda, M. K.; Wisniewski, S. R.; Eastgate, M. D.; Engle, K. M. Nickel-catalyzed 1,2-diarylation of simple alkenyl amides. J. Am. Chem. Soc. 2018, 140, 17878-17883. (m) Campbell, M. W.; Compton, J. S.; Kelly, C. B.; Molander, G. A. Three-component olefin dicarbofunctionalization enabled by nickel/photoredox dual catalysis. J. Am. Chem. Soc. 2019, 141, 20069-20078. (n) Derosa, J.; Kang, T.; Tran, V. T.; Wisniewski, S. R.; Karunananda, M. K.; Jankins, T. C.; Xu, K. L.; Engle, K. M. NickelCatalyzed 1,2-Diarylation of Alkenyl Carboxylates A Gateway to 1,2,3Trifunctionalized Building Blocks. Angew. Chem. Int. Ed. 2020, 59, 12011205. (o) Mega, R. S.; Duong, V. K.; Noble, A.; Aggarwal, V. K. Decarboxylative conjunctive cross-coupling of vinyl boronic esters using metallaphotoredox catalysis. Angew. Chem. Int. Ed. 2020, 59, 4375-4379. (p) Liu, L.; Lee, W.; Youshaw, C. R.; Yuan, M.; Geherty, M. B.; Zavalij, P. Y.; Gutierrez, O. Fe-catalyzed three-component dicarbofunctionalization of unactivated alkenes with alkyl halides and Grignard reagents. Chem. Sci. 2020, 11, 8301-8305.

(3) Sandford, C.; Aggarwal, V. K. Stereospecific functionalizations and transformations of secondary and tertiary boronic esters. Chem. Commun. 2017, 53, 5481-5494.

(4) Frisch, A. C.; Beller, M. Catalysts for cross-coupling reactions with non-activated alkyl halides. Angew. Chem. Int. Ed. 2005, 44, 674-688.
(5) Ariafard, A.; Lin, Z. Understanding the relative easiness of oxidative addition of aryl and alkyl halides to palladium(0). Organometallics 2006, $25,4030-4033$

(6) Terao, J.; Kato, Y.; Kambe. N. Titanocene-catalyzed regioselective alkylation of styrenes with Grignard reagents using $\beta$-bromoethyl ethers, thioethers, or amines. Chem. Asian J. 2008, 3, 1472-1478.

(7) Mizutani, K.; Shinokubo, H.; Oshima. K. Cobalt-catalyzed threecomponent coupling reaction of alkyl halides, 1,3-dienes, and trimethylsilylmethylmagnesium chloride. Org. Lett. 2003, 5, 3959-3961.

(8) (a) Phapale, V. B.; Buñuel, E.; García-Iglesias, M.; Cárdenas. D. J. $\mathrm{Ni}$-catalyzed cascade formation of $\mathrm{C}\left(\mathrm{sp}^{3}\right)-\mathrm{C}\left(\mathrm{sp}^{3}\right)$ bonds by cyclization and cross-coupling reactions of iodoalkanes with alkyl zinc halides. Angew. Chem. Int. Ed. 2007, 46, 8790-8795. For a two-component dialkylation method under reductive conditions, see: (b) Kuang, Y.; Wang, X.; Anthony, D.; Diao, T. Ni-catalyzed two-component reductive dicarbofunctionalization of alkenes via radical cyclization. Chem. Commun. 2018, 54, 25582561.

(9) Derosa, J.; van der Puyl, V. A.; Tran, V. T.; Liu, M.; Engle, K. M Directed nickel-catalyzed 1,2-dialkylation of alkenyl carbonyl compounds. Chem. Sci. 2018, 9, 5278-5283.

(10) For selected reviews and examples, see: (a) Weix, D. J. Methods and mechanisms for cross-electrophile coupling of $\mathrm{Csp}^{2}$ halides with alkyl electrophiles. Acc. Chem. Res. 2015, 48, 1767-1775. (b) Gu, J.; Wang, X.; Xue, W.; Gong, H. Nickel-catalyzed reductive coupling of alkyl halides with other electrophiles: concept and mechanistic considerations. Org. Chem. Front. 2015, 2, 1411-1421. (c) Knappke, C. E. I.; Grupe, S.; Gärtner, D.; Corpet, M.; Gosmini, C.; Wangelin, A. J. von. Reductive crosscoupling reactions between two electrophiles. Chem. Eur. J. 2014, 20, 6828-6842. (d) Everson, D. A.; Shrestha, R.; Weix, D. J. Nickel-Catalyzed Reductive Cross-Coupling of Aryl Halides with Alkyl Halides. J. Am. Chem. Soc. 2010, 132, 920-921. (e) Yu, X.; Yang, T.; Wang, S.; Xu, H.; Gong, H. Nickel-catalyzed reductive cross-coupling of unactivated alkyl halides. Org. Lett. 2011, 13, 2138-2141. (f) Everson, D. A.; Jones, B. A.; Weix, D. J. Replacing conventional carbon nucleophiles with electrophiles: nickel-catalyzed reductive alkylation of aryl bromides and chlorides $J$. Am. Chem. Soc. 2012, 134, 6146-6159. (g) Biswas, S.; Weix, D. J. Mechanism and selectivity in nickel-catalyzed cross-electrophile coupling of aryl halides with alkyl halides. J. Am. Chem. Soc. 2013, 135, 16192-16197. (h) Arendt, K. M.; Doyle, A. G. Dialkyl ether formation by nickel-catalyzed cross-coupling of acetals and aryl iodides. Angew. Chem. Int. Ed. 2015, 54, 9876-9880. (i) Kadunce, N.; Reisman, S. E. Nickel-catalyzed asymmetric reductive cross-coupling between heteroaryl iodides and $\alpha$-chloronitriles. $J$. Am. Chem. Soc. 2015, 137, 10480-10483. (j) Wang, X.; Wang, S.; Xue, W.; Gong, H. Nickel-catalyzed reductive coupling of aryl bromides with tertiary alkyl halides. J. Am. Chem. Soc. 2015, 137, 11562-11565. (k) Konev, M. O.; Hanna, L. E.; Jarvo, E. R. Intra- and intermolecular nickelcatalyzed reductive cross-electrophile coupling reactions of benzylic esters with aryl halides. Angew. Chem. Int. Ed. 2016, 55, 6730-6733.

(11) (a) García-Domínguez, A.; Li, Z.; Nevado, C. Nickel-catalyzed reductive dicarbofunctionalization of alkenes. J. Am. Chem. Soc. 2017, 139, 6835-6838. (b) Zhao, X.; Tu, H. Y.; Guo, L.; Zhu, S.; Qing, F. L.; Chu, L. Intermolecular selective carboacylation of alkenes via nickel-catalyzed reductive radical relay. Nat. Commun. 2018, 9, 3488. (c) Shu, W.; GarcíaDomínguez, A.; Quirós, M. T.; Mondal, R.; Cárdenas, D. J.; Nevado, C. Nicatalyzed reductive dicarbofunctionalization of nonactivated alkenes: scope and mechanistic insights. J. Am. Chem. Soc. 2019, 141, 13812-13821. (d) Yang, T.; Chen, X.; Rao, W.; Koh, M. J. Broadly applicable directed catalytic reductive difunctionalization of alkenyl carbonyl compounds. Chem. 2020, 6, 738-751. (e) Tu, H. Y.; Wang, F.; Huo, L. P.; Li, Y. B.; Zhu, S. Q.; Zhao, X.; Li, H.; Qing, F. L.; Chu, L. Enantioselective three-component fluoroalkylarylation of unactivated olefins through nickel-catalyzed crosselectrophile coupling. J. Am. Chem. Soc. 2020, 142, 9604-9611. (f) Wei, X.; Shu, W.; García-Domínguez, A.; Merino, E.; Nevado. C. Asymmetric Ni-catalyzed radical relayed reductive coupling. J. Am. Chem. Soc. 2020, 142, 13515-13522. (g) Sun, S.; Duan, Y.; Mega, R. S.; Somerville, R. J.; Martin, R. Site-selective 1,2-dicarbofunctionalization of vinyl boronates through dual catalysis. Angew. Chem. Int. Ed. 2020, 59, 4370-4374.

(12) (a) Cornella, J.; Edwards, J. T.; Qin, T.; Kawamura, S.; Wang, J.; Pan, C.; Gianatassio, R.; Schmidt, M.; Eastgate, M. D.; Baran. P. S. Practical Ni-catalyzed aryl-alkyl cross-coupling of secondary redox-active esters. J. Am. Chem. Soc. 2016, 138, 2174-2177. (b) Murarka, S. N(Acyloxy)phthalimides as redox-active esters in cross-coupling reactions. Adv. Synth. Catal. 2018, 360, 1735-1753.

(13) (a) Fu, M.; Shang, R.; Zhao, B.; Wang, B.; Fu, Y. Photocatalytic decarboxylative alkylations mediated by triphenylphosphine and sodium iodide. Science 2019, 363, 1429-1434. (b) Tlahuext-Aca, A.; Garza- 
Sanchez, R. A.; Glorius. F. Multicomponent oxyalkylation of styrenes enabled by hydrogen bond-assisted photoinduced electron transfer. Angew. Chem. Int. Ed. 2017, 56, 3708-3711. (c) Qin, T.; Cornella, J.; Li, C.; Malins, L. R.; Edwards, J. T.; Kawamura, S.; Maxwell, B. D.; Eastgate, M. D.; Baran, P. S. A general alkyl-alkyl cross-coupling enabled by redox-active esters and alkylzinc reagents. Science 2016, 352, 801-805. (d) Lu, X.; Wang, X.; Gong, T.; Pi, J.; He, S.; Fu, Y. Nickel-catalyzed allylic defluorinative alkylation of trifluoromethyl alkenes with reductive decarboxylation of redox-active esters. Chem. Sci. 2019, 10, 809-814.

(14) Huang, H. M.; Koy, M.; Serrano, E.; Pflüger, P. M.; Schwarz, J. L.; Glorius, F. Catalytic radical generation of $\pi$-allylpalladium Complexes. Nat. Catal. 2020, 3, 393-400.

(15) Ackerman, L. K. G.; Lovell, M. M.; Weix, D. J. Multimetallic catalysed cross-coupling of aryl bromides with aryl triflates. Nature 2015, 524, $454-457$.

(16) Komeyama, K.; Michiyuki, T.; Osaka, I. Nickel/cobalt-catalyzed $\mathrm{C}\left(\mathrm{sp}^{3}\right)-\mathrm{C}\left(\mathrm{sp}^{3}\right)$ cross-coupling of alkyl halides with alkyl tosylates. ACS Catal. 2019, 9, 9285-9291.

(17) Huihui, K. M. M.; Caputo, J. A.; Melchor, Z.; Olivares, A. M.; Spiewak, A. M.; Johnson, K. A.; DiBenedetto, T. A.; Kim, S.; Ackerman, L. K. G.; Weix, D. J. Decarboxylative cross-electrophile coupling of Nhydroxyphthalimide esters with aryl iodides. J. Am. Chem. Soc. 2016, 138 , 5016-5019.

(18) Huang, L.; Olivares, A. M.; Weix, D. J. Reductive decarboxylative alkynylation of $N$-hydroxyphthalimide esters with bromoalkynes. Angew. Chem. Int. Ed. 2017, 56, 11901-11905.

(19) Zhao, Y.; Truhlar, D. G. Density functionals with broad applicability in chemistry. Acc. Chem. Res. 2008, 41, 157-167.

(20) (a) Pan, X.; Fang, C.; Fantin, M.; Malhotra, N.; So, W. Y.; Peteanu, L. A.; Isse, A. A.; Gennaro, A.; Liu, P. ; Matyjaszewski, K. Mechanism of photoinduced metal-free atom transfer radical polymerization: experimental and computational studies. J. Am. Chem. Soc. 2016, 138, 2411-2425. (b) Yuan, M.; Song, Z.; Badir, S. O.; Molander, G. A.; Gutierrez, O. On the nature of $\mathrm{C}\left(\mathrm{sp}^{3}\right)-\mathrm{C}\left(\mathrm{sp}^{2}\right)$ bond formation in nickel-catalyzed tertiary radical cross-couplings: a case study of Ni/photoredox catalytic cross-coupling of alkyl radicals and aryl halides. J. Am. Chem. Soc. 2020, 142, 7225-7234. (c) Chen, J.; Xu, M.; Yu, S.; Xia, Y.; Lee, S. Nickelcatalyzed claisen condensation reaction between two different amides. $\mathrm{Org}$. Lett. 2020, 22, 2287-2292.

(21) (a) Saveant, J. M. A simple model for the kinetics of dissociative electron transfer in polar solvents. Application to the homogeneous and heterogeneous reduction of alkyl halides. J. Am. Chem. Soc. 1987, 109, 67886795. (b) Fang, C.; Fantin, M.; Pan, X.; Fiebre, K.; Coote, M. L.; Matyjaszewski, K.; Liu, P. Mechanistically guided predictive models for ligand and initiator effects in copper-catalyzed atom transfer radical polymerization (Cu-ATRP). J. Am. Chem. Soc. 2019, 141, 7486-7497.

(22) (a) Lan, Y.; Wheeler, S. E.; Houk, K. N. Extraordinary difference in reactivity of ozone (OOO) and sulfur dioxide (OSO): A theoretical study. J. Chem. Theory Comput. 2011, 7, 2104-2111. (b) Liu, S.; Lei, Y.; Qi, X.; Lan, Y. Reactivity for the Diels-Alder reaction of cumulenes: A distortioninteraction analysis along the reaction pathway. J. Phys. Chem. A 2014, $118,2638-2645$.

(23) Manolikakes, G.; Dong, Z. B.; Mayr, H.; Li, J. S.; Knochel, P. Negishi cross-couplings compatible with unprotected amide functions. Chem. Eur. J. 2009, 15, 1324-1328.

(24) Haginaka, J.; Yasuda, H.; Uno, T.; Nakagawa, T. Alkaline degradation and determination by high-performance liquid chromatography. Chem. Pharm. Bull. 1984, 32, 2752-2758.

(25) Blankson, G. A.; Parhi, A. K.; Kaul, M.; Pilch, D. S.; LaVoie, E. J. Advances in the structural studies of antibiotic potentiators against Escherichia coli. Bioorg. Med. Chem. 2019, 27, 3254-3278.

(26) Yamauchi, S.; Shoji, Y.; Nishimoto, A.; Uzura, M.; Nishiwaki, H.; Nishi, K.; Sugahara, T. Design of 92 new 9-norlignan derivatives and their effect on cell viabilities of cancer and insect cells. J. Agric. Food Chem. 2019, 67, 7880-7885.

(27) (a) Geist, E.; Kirschning, A.; Schmidt, T. $\mathrm{sp}^{3}$-sp $\mathrm{sp}^{3}$ Coupling reactions in the synthesis of natural products and biologically active molecules. Nat. Prod. Rep. 2014, 31, 441-448. (b) Lovering, F.; Bikker, J.; Humblet, C. Escape from flatland: increasing saturation as an approach to improving clinical success. J. Med. Chem. 2009, 52, 6752-6756. 